\title{
Designing Active Disturbance Rejection Control of Permanent Magnetic Spherical Actuator Based on Nonlinear Extended State Observer
}

\author{
Xiwen Guo ${ }^{1,2, *}$, Xianda Liao, ${ }^{1,2}$, Qunjing Wang, ${ }^{2,3}$, Yan Wen ${ }^{4}$, Tao Wu ${ }^{1,2}$ \\ ${ }^{1}$ School of Electrical Engineering and Automation, Anhui University, \\ Hefei, 230601, China \\ ${ }^{2}$ National Engineering Laboratory of Energy-Saving Motor \& Control Technology, Anhui University, \\ Hefei, 230601, China \\ ${ }^{3}$ Anhui Key Laboratory of Industrial Energy-Saving and Safety, Anhui University, \\ Hefei, 230601, China \\ ${ }^{4}$ School of Internet, Anhui University, \\ Hefei, 230601, China \\ xw_guo@ahu.edu.cn
}

\begin{abstract}
Aiming at the problems of low control precision and poor anti-disturbances ability of permanent magnet spherical actuator (PMSA), an active disturbance rejection trajectory tracking control method based on nonlinear extended state observer (NESO) is proposed in this paper. Firstly, the multivariable and strong coupling mathematical model of the PMSA is obtained by rigid body rotation coordinate transformation and the Lagrange dynamic equation. Then, the tracking differentiator is used to reduce the influence caused by the expected trajectory jump. In addition, NESO is designed to observe and calculate coupling of the system, external disturbance, and model error online. Finally, the nonlinear control law is used to compensate for the observed disturbance, and the stability is proved based on the Lyapunov equation. Simulation and experimental results show that the proposed control scheme can achieve decoupling and tracking control of the complex system in the presence of model error, random disturbance, and other uncertain factors, has good control accuracy and response speed, and has strong robustness to uncertain disturbances.
\end{abstract}

Index Terms-Permanent magnet spherical actuator; Active disturbance rejection control; Trajectory tracking; Nonlinear extended state observer.

\section{INTRODUCTION}

PMSA has the advantages of simple structure, small volume, high positioning accuracy, fast response speed, and good dynamic and static performance. Because it can realize the motion of multiple degrees of freedom in the narrow joint space and directly drive the load, thus simplifying the motion control system. Therefore, once proposed, it has attracted much attention and has been widely used in the fields of high-end equipment demand, such as multi-dimensional precision machining [1], radar tracking system [2], human

Manuscript received 15 November, 2021; accepted 12 January, 2022

This research was supported by the National Natural Science Foundation of China under Grants Nos. 51637001 and 51307001, the Natural Science Foundation of Anhui Province under Grant No. 2008085ME156, the Young Core Teacher Program of Anhui University under Grant No. J01005126. prosthesis [3], new energy vehicle [4], etc.

However, a permanent magnet spherical actuator (PMSA) is a typical multivariable, coupled, time-varying nonlinear system. Therefore, in the process of body design [5], magnetic field and torque analysis [6], [7], dynamics analysis [8], and PMSA motion control [9]-[11], more factors should be considered than the traditional motor.

To make PMSA from theoretical research to practical application, many control schemes have been put forward. The more classic is to apply the traditional Proportional Integral Derivative (PID) control to PMSA [12], [13]. Although the PID control method is simple in design and easy to implement, it is difficult to guarantee the accuracy of this control method when there are external disturbances and uncertain factors, and it is difficult for simple PID control to have a good effect on the complex nonlinear system of PMSA. To reduce the influence of external disturbances and uncertainties on the control system, the sliding mode control is applied to many fields, such as the permanent magnet synchronous generator [14], and so is the PMSA [15], [16]. Although the scheme can overcome the uncertainty of the system and has certain robustness to external disturbances. However, due to the special structure of the PMSA and the problem of buffeting when the state trajectory reaches the sliding mode surface, it is difficult to guarantee good dynamic performance by using this controller. Reference [17] attempts to use an adaptive iterative method to control PMSA. This method does not consider the coupling effect of the system and has certain requirements for the calculation speed of iterative tools, so it is not suitable for practical application. Although the computational torque method [18]-[20] can be used to decouple the system, it does not have a good suppression effect on the external disturbance of uncertainty. In addition, some scholars proposed to introduce a neural network with good robustness and decoupling to solve the above problems. However, the scheme requires a lot of calculation, so it is difficult to ensure the real-time 
control of the scheme [21]-[23]. In addition, a parameter identification method of the Coulomb friction model is proposed for nonlinear friction, and it is compensated in the closed-loop control [24]. However, the distribution of friction torque field needs to be obtained through complex simulation and experiment, which is also difficult to ensure real-time performance. To obtain cascaded 6-DOF motion, a robust terminal sliding mode control method is proposed to precisely manipulate the orientation of the spherical motor in the motion frame [25]. However, some parameter design is relatively troublesome and it is difficult to take into account both tracking error and chatter.

Considering all these issues, it may be a better choice to apply active disturbance rejection control (ADRC) technology to decoupling and tracking control of PMSA given uncertain factors, such as model error and random disturbance. ADRC evolved from PID control [26]. It inherits the essence of the classical PID controller and introduces state observer technology based on modern control theory. The uncertain and complex factors, which are coupled by the internal dynamics without modeling, external disturbances, and complex nonlinear factors, are regarded as the total disturbance of the system. The extended observer is used to estimate the total disturbance online and compensate in the feedback control link, so that the multivariable system can be decoupled easily. Moreover, it does not need the mathematical model of the controlled object. It is very powerful to suppress the interference and noise and has a small amount of calculation. It can significantly improve the real-time performance of the system. At present, more in-depth research has been performed in the fields of motion control system [27], [28], satellite control system [29], robot motion control system [30], and four-rotor control system [31]. Among them, reference [32] has tried to apply ADRC technology to the PMSA field for the first time, and has obtained certain anti-interference ability and decoupling effect. However, because the design scheme is essentially PID control based on the linear extended state observer (LESO), the control accuracy is still not guaranteed. Therefore, based on the ADRC model, this paper uses a nonlinear extended state observer (NESO) to observe the internal coupling and external disturbance of the PMSA system online. Therefore, the control scheme in this paper has the following advantages:

1. The ADRC control scheme proposed in this paper uses a nonlinear structure, which can achieve better control accuracy when applied to the PMSA;

2. The improved NESO only contains a few parameters, which greatly simplifies the process of parameter setting; 3. The control strategy in this paper can effectively eliminate the influence of internal dynamics and external uncertain disturbance in PMSA and has strong decoupling and robustness. In addition, less calculation makes the control scheme have better real-time performance, better static performance, and dynamic performance.

The contents of this paper are arranged as follows. Section II introduces the basic structure and dynamic model of the PMSA. In Section III, an ADRC scheme based on a nonlinear state observer is proposed. In Sections IV and V, simulation and experimental studies are carried out to verify the feasibility and superiority of this method. Finally, the idea developed is summarized in Section VI.

\section{BASIC Structure AND DyNAMIC MODELling OF PMSA}

\section{A. Structure of PMSA}

Reference [33] introduces the detailed structure of the PMSA in this paper. The structure of the PMSA is shown in Fig. 1(a), and the simulation model is shown in Fig. 1(b). It is composed of rotor, stator shell, permanent magnet, coil, and output shaft. The stator is designed as two-hemispherical shell structure. The stator coil is divided into two layers, 12 in each layer, which are evenly and symmetrically fixed on the two hemispherical shells. The spherical rotor is fixed to the spherical shell of the stator. There are four layers of alternating polarity, symmetrical, and evenly distributed 40 permanent magnets in the rotor. The rotor is supported by a ring at the bottom. Due to the influence of the motor structure, the rotor output shaft is limited, and the attitude angle can only change in a certain range. The rotation angle of the $\mathrm{x}$-axis $\alpha$ and the $\mathrm{y}$-axis $\beta$ is from $-37.5^{\circ}$ to $37.5^{\circ}$, and the rotation angle $\gamma$ of the $\mathrm{z}$-axis is not affected, which is still from $0^{\circ}$ to $360^{\circ}$. The schematic diagram of the rotation mode is shown in Fig. 1(c).

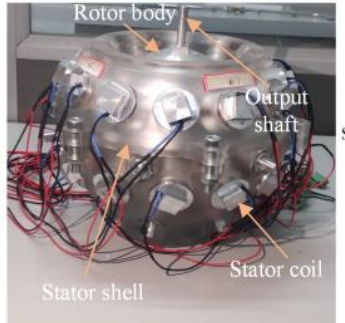

(a)

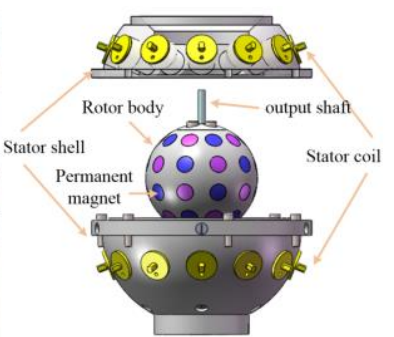

(b)

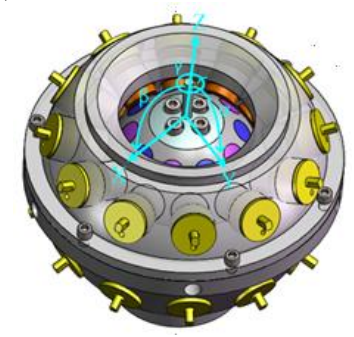

(c)

Fig. 1. (a) Prototype of PMSA; (b) Physical model of PMSA; (c) Rotation mode of the rotor.

\section{B. Dynamic Model of PMSA}

Since the rotor of permanent magnet synchronous motor is a rigid body, the kinematic model of PMSA can be established according to the transformation of the coordinate of the rotation of the rigid body and the Lagrange dynamic equation [34]

$$
M(\theta) \ddot{\theta}+C(\theta, \dot{\theta}) \dot{\theta}+\Delta(\theta, \dot{\theta})+d=T-T_{f}
$$

where $\Delta(\boldsymbol{\theta}, \dot{\boldsymbol{\theta}})$ is the model error, $d$ is the external disturbance, $\boldsymbol{T}_{f}$ is the friction force encountered in the process of rotor motion, $\boldsymbol{\theta}=\left[\begin{array}{lll}\alpha & \beta & \gamma\end{array}\right]^{T}$ is the angular displacement, $\boldsymbol{T}=\left[\begin{array}{lll}\boldsymbol{T}_{x} & \boldsymbol{T}_{\boldsymbol{y}} & \boldsymbol{T}_{z}\end{array}\right]^{\boldsymbol{T}}$ is the PMSA control torque, $\boldsymbol{M}$ is the inertial matrix, and $\boldsymbol{C}$ is the Coriolis matrix. 
The components of $\boldsymbol{M}$ and $\boldsymbol{C}$ can be expressed as follows:

$$
\begin{aligned}
& \left\{\begin{array}{l}
M_{11}=I_{x} \cos ^{2} \beta \cos ^{2} \gamma+I_{y} \cos ^{2} \beta \sin ^{2} \gamma+I_{z} \sin ^{2} \beta, \\
M_{12}=\left(I_{x}-I_{y}\right) \cos \beta \sin \gamma \cos \gamma, \\
M_{13}=M_{31}=I_{z} \sin \beta,
\end{array}\right. \\
& \left\{\begin{array}{l}
M_{21}=\left(I_{x}-I_{y}\right) \cos \beta \sin \gamma \cos \gamma \\
M_{22}=I_{x} \sin ^{2} \gamma+I_{y} \cos ^{2} \gamma \\
M_{23}=0
\end{array}\right. \\
& \left\{\begin{array}{l}
M_{31}=I_{z} \sin \beta \\
M_{32}=0 \\
M_{33}=I_{z}
\end{array}\right. \\
& C_{11}=\left(I_{z}-I_{x} \sin ^{2} \gamma-I_{y} \sin ^{2} \gamma\right) \dot{\beta} \sin \beta \cos \beta+ \\
& +\left(I_{y}-I_{x}\right) \cos ^{2} \beta \sin \gamma \cos \gamma, \\
& \left\{C_{12}=\left(I_{x} \cos ^{2} \gamma+I_{y} \sin ^{2} \gamma\right) \dot{\gamma} \cos \beta+I_{z} \cos \beta(\dot{\gamma}+\right. \\
& +\dot{\alpha} \sin \beta)-\left(I_{x} \cos ^{2} \gamma+I_{y} \sin ^{2} \gamma\right) \dot{\alpha} \sin \beta \cos \beta, \\
& C_{13}=-\dot{\beta} \cos \beta\left(I_{x} \cos ^{2} \gamma+I_{y} \sin ^{2} \gamma\right), \\
& C_{21}=\left(I_{y} \cos ^{2} \gamma+I_{x} \sin ^{2} \gamma\right)(-\dot{\gamma} \cos \beta)+\left(I_{x} \cos ^{2} \gamma+\right. \\
& \left.+I_{y} \sin ^{2} \gamma\right) \dot{\alpha} \sin \beta \cos \beta-I_{z} \dot{\gamma} \cos \beta-I_{z} \dot{\alpha} \sin \beta \cos \beta, \\
& C_{22}=\left(I_{x}-I_{y}\right) \dot{\gamma} \sin \gamma \cos \gamma, \\
& C_{23}=\dot{\alpha} \cos \beta\left(I_{x} \cos ^{2} \gamma+I_{y} \sin ^{2} \gamma\right) \text {, } \\
& \left\{\begin{aligned}
C_{31} & =\left(I_{x}-I_{y}\right) \dot{\alpha} \sin \gamma \cos \gamma \cos ^{2} \beta+ \\
& +\left(I_{x} \cos ^{2} \gamma+I_{y} \sin ^{2} \gamma+I_{z}\right) \dot{\beta} \cos \beta, \\
C_{32} & =\left(I_{x}-I_{y}\right) \dot{\alpha} \sin \gamma \cos \gamma \cos ^{2} \beta+ \\
& +\left(I_{x} \cos ^{2} \gamma+I_{y} \sin ^{2} \gamma++I_{z}\right) \dot{\beta} \cos \beta, \\
C_{33}= & 0 .
\end{aligned}\right.
\end{aligned}
$$

Thus, the control model is obtained as follows

$$
\ddot{\boldsymbol{\theta}}=M^{-1}(\boldsymbol{\theta})\left(\boldsymbol{T}-\boldsymbol{C}(\boldsymbol{\theta}, \dot{\boldsymbol{\theta}}) \dot{\boldsymbol{\theta}}-\Delta(\boldsymbol{\theta}, \dot{\boldsymbol{\theta}})-d-T_{f}\right)
$$

\section{DESIGN OF ADRC}

In general, ADRC consists of three components: a tracking differentiator, a nonlinear state feedback controller, and a nonlinear extended state observer. The ADRC flow chart for PMSA is shown in Fig. 2 and is described below.

According to the flow chart, build the ADRC control module for PMSA, as follows.

\section{A. Tracking Differentiator}

The essence of traditional PID control is to eliminate error based on error feedback. However, the feedback is 0 at the initial time, which makes it easy to produce overshoot. In this paper, a nonlinear differential tracker is used to solve the overshoot problem. Assuming that the expected trajectory of the three rotation angles is $\boldsymbol{V}$, the expression of the tracking differentiator is as follows

$$
\left\{\begin{array}{l}
\dot{V}_{1}=V_{2}, \\
\dot{\boldsymbol{V}}_{2}=f s t\left(\boldsymbol{V}_{1}-\boldsymbol{V}, \boldsymbol{V}_{2}, \varphi, h\right),
\end{array}\right.
$$

where $\boldsymbol{V}=\left[\begin{array}{lll}\boldsymbol{V}_{x} & \boldsymbol{V}_{y} & \boldsymbol{V}_{z}\end{array}\right]^{T} . h$ is the sampling time, which should not be too small. Otherwise, although the tracking accuracy can be improved, the noise may also be amplified. $\varphi$ is to determine the tracking speed. The $f_{s t}$ (.) function is the synthesis function of the fastest control, which is used to solve the problem of slow tracking speed when the overshoot is removed.

The expression of the $f s t$ (.) function is as follows

$$
f s t\left(\boldsymbol{V}_{1}-\boldsymbol{V}, \boldsymbol{V}_{2}, \varphi, h\right)= \begin{cases}-\varphi \operatorname{sgn}(k), & |k|>d, \\ -\varphi k / d, & |k| \leq d,\end{cases}
$$

where:

$$
\begin{gathered}
k= \begin{cases}\boldsymbol{V}_{2}+\left(m_{0}-d\right) \operatorname{sgn}(n) / 2, & |n|>d_{0}, \\
\boldsymbol{V}_{2}+y / h, & |n| \leq d_{0},\end{cases} \\
\left\{\begin{array}{l}
d=\varphi h, \\
d_{0}=d h, \\
n=\boldsymbol{V}_{1}-\boldsymbol{V}+h \boldsymbol{V}_{2}, \\
m_{0}=\sqrt{d_{2}+8 \varphi|y|} .
\end{array}\right.
\end{gathered}
$$

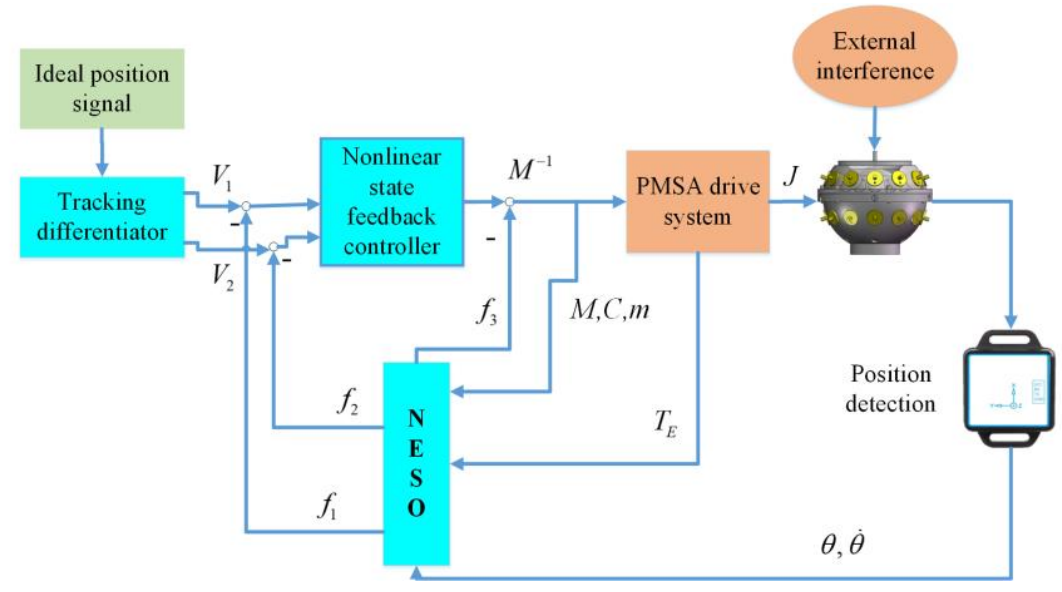

Fig. 2. PMSA ADRC flow chart. 


\section{B. NESO}

If all the effects are regarded as the total disturbance $m$, according to the dynamic control model of (8), the dynamic model of rotation angle is as follows

$$
\ddot{\boldsymbol{\theta}}=\boldsymbol{M}^{-1}(\boldsymbol{\theta})\left(\boldsymbol{T}_{E}-\boldsymbol{C}(\boldsymbol{\theta}, \dot{\boldsymbol{\theta}}) \dot{\boldsymbol{\theta}}-m\right)
$$

According to (8), the control model of rotation angle is as follows:

$$
\left\{\begin{array}{l}
\dot{x_{1}}=x_{2}, \\
\dot{x}_{2}=M_{1}^{-1}\left(x_{1}\right)\left[T_{E}-C\left(x_{1}, x_{2}\right) x_{2}-m\right], \\
y=x_{1}
\end{array}\right.
$$

where $\boldsymbol{x}_{1}=\boldsymbol{\theta}$ and $\boldsymbol{x}_{2}=\dot{\boldsymbol{\theta}}$.

Therefore, according to the characteristics of the PMSA, a third-order NESO is designed to observe the coupling and disturbance of the system. The extended nonlinear observer is expressed as seen in (15):

$$
\left\{\begin{array}{l}
\boldsymbol{e}=\boldsymbol{f}_{1}-\dot{\boldsymbol{f}}_{1}, \\
\dot{\boldsymbol{f}}_{1}=\boldsymbol{f}_{2}-\boldsymbol{b} \operatorname{fal}\left(\boldsymbol{e} / \boldsymbol{b}^{2}, \partial_{1}, \delta\right) \\
\dot{\boldsymbol{f}}_{2}=\boldsymbol{f}_{3}-\operatorname{fal}\left(\boldsymbol{e} / \boldsymbol{b}^{2}, \partial_{1}, \delta\right)+\boldsymbol{M}^{-1}(\boldsymbol{\theta}) \boldsymbol{T}_{\boldsymbol{E}} \\
\dot{\boldsymbol{f}}_{3}=-\boldsymbol{b}^{-1} \operatorname{fal}\left(\boldsymbol{e} / \boldsymbol{b}^{2}, \partial_{2}, \delta\right),
\end{array}\right.
$$

where $\boldsymbol{f}_{1}=\left[\begin{array}{lll}f_{1 \mathrm{x}} & f_{1 \mathrm{y}} & f_{1 \mathrm{z}}\end{array}\right]^{T}, \quad \boldsymbol{f}_{2}=\left[\begin{array}{lll}f_{2 \mathrm{x}} & f_{2 \mathrm{y}} & f_{2 \mathrm{z}}\end{array}\right]^{T}$, $\boldsymbol{f}_{3}=\left[\begin{array}{lll}f_{3 \mathrm{x}} & f_{3 \mathrm{y}} & f_{3 \mathrm{z}}\end{array}\right]^{T}$ are the values observed by NESO, with the initial value of $f_{1}$ being set to 0 ; $\boldsymbol{e}=\left[\begin{array}{lll}e_{x} & e_{y} & e_{z}\end{array}\right]^{T}, \boldsymbol{b}=\left[\begin{array}{lll}b_{x} & b_{y} & b_{z}\end{array}\right]^{T}$ are the parameters to be set by the observer, and $f a l(\boldsymbol{e}, \partial, \delta)$ is a nonlinear function, and its expression is as follows

$$
f a l(\boldsymbol{e}, \partial, \delta)=\left\{\begin{array}{cl}
\boldsymbol{e} / \delta^{l-\partial}, & |\boldsymbol{e}| \leq \delta, \\
|\boldsymbol{e}|^{2} \operatorname{sgn}(\boldsymbol{e}), & |\boldsymbol{e}|>\delta,
\end{array}\right.
$$

where $\partial$ is the parameter of the nonlinear function, which is selected in (15), $\partial_{1}=\left[\begin{array}{lll}0.5 & 0.5 & 0.5\end{array}\right], \partial_{2}=\left[\begin{array}{lll}0.5 & 0.5 & 0.5\end{array}\right]$.

\section{Nonlinear State Feedback Controller}

After the tracking differentiator and the NESO are designed, in order to have better control performance, the nonlinear state feedback controller is used

$$
\boldsymbol{T}_{0}=K_{p} * f a l\left(\boldsymbol{e}_{1}, \partial_{1}, \delta\right)+K_{d} * f a l\left(\boldsymbol{e}_{2}, \partial_{2}, \delta\right)
$$

where $e_{1}=V_{1}-f_{1}$ and $e_{2}=V_{2}-f_{2}$.

To compensate for the total disturbance observed $f_{3}$, the following results can be obtained

$$
\boldsymbol{T}_{\boldsymbol{E}}=\boldsymbol{T}_{0}-\boldsymbol{M}(\boldsymbol{\theta}) * \boldsymbol{f}_{3}
$$

\section{Power on Strategy Design System Current Calculation}

As described above, after designing the relevant modules of $y$-axis and z-axis, respectively, how to calculate the required current of the system through torque can be considered. Because the PMSA used in this paper is an air coil, the interaction of a single rotor pole can be expressed by the following equation without considering the saturation effect of magnetic field

$$
\boldsymbol{T}_{i j}=f\left(\boldsymbol{\theta}_{i j}\right) \frac{s_{r i} \times s_{s j}}{\left|s_{r i} \times s_{s j}\right|} N i,
$$

where $i$ is the number of permanent magnets, the value ranges from 1 to 40. $j$ is the number of coils with a value ranging from 1 to 24. $s_{r i}, s_{s j}$ is the vector position of rotor permanent magnet and stator permanent magnet, $N i$ is the number of ampere turns of coil, its value is 2400. $f(\boldsymbol{\theta})$ is the moment angle characteristic of the single stator and the rotor pole action, which can be obtained by the fitting method, and $\boldsymbol{\theta}$ is the rotation angle.

From (19), when all the stator and rotor poles of the PMSA interact, the resultant torque of the whole motor is

$$
\boldsymbol{T}_{\boldsymbol{E}}=\sum_{i=1}^{40} \sum_{j=1}^{24} f\left(\boldsymbol{\theta}_{i j}\right) \frac{s_{r i} \times s_{s j}}{\left|s_{r i} \times s_{s j}\right|} N i .
$$

Equation (20) is written in the form of a matrix

$$
\boldsymbol{T}_{\boldsymbol{E}}=\left[\begin{array}{c}
T_{x} \\
T_{y} \\
T_{z}
\end{array}\right]=\left[\begin{array}{cccc}
f x_{1} & f x_{2} & \ldots & f x_{24} \\
f y_{1} & f y_{2} & \ldots & f y_{24} \\
f z_{1} & f z_{2} & \ldots & f z_{24}
\end{array}\right] \times\left[\begin{array}{c}
J_{1} \\
J_{2} \\
\vdots \\
J_{24}
\end{array}\right]=\boldsymbol{A} \times \boldsymbol{J}
$$

where $f x_{i}, f y_{i}, f z_{i}$ is the torque component along the $x, y, z$ direction generated by the unit current, matrix $\boldsymbol{A}$ changes with the rotation angle. $J_{i}$ is the current of the $i^{\text {th }}$ coil.

According to (21), the required current of each coil in the system is as follows

$$
J=A^{-1} \times T_{E}
$$

\section{E. Stability Proof of NESO}

To prove the stability proposed above, we need to use two continuous positive definite functions $U, W$.

Firstly, there exists a positive definite matrix in the following form

$$
\boldsymbol{Q}=\left[\begin{array}{lll}
-a_{1} & 1 & 0 \\
-a_{2} & 0 & 1 \\
-a_{3} & 0 & 0
\end{array}\right]
$$

Let the positive definite matrix $\boldsymbol{P}$ be the solution of the 
Lyapunov matrix equation $\boldsymbol{P} \boldsymbol{Q}+\boldsymbol{Q}^{T} \boldsymbol{P}=-\boldsymbol{E}$, where $E$ represents the third-order identity matrix. Define the functions $U$ and $W$ as:

$$
\begin{aligned}
& U(\boldsymbol{\mu})=\langle\boldsymbol{P} \boldsymbol{\mu} \quad \boldsymbol{\mu}\rangle, \forall \boldsymbol{\mu} \in \boldsymbol{R}^{3}, \\
& W(\boldsymbol{\mu})=\langle\boldsymbol{\mu} \quad \boldsymbol{\mu}\rangle, \forall \boldsymbol{\mu} \in \boldsymbol{R}^{3} .
\end{aligned}
$$

So:

$$
\begin{gathered}
\lambda_{\text {min }}(\boldsymbol{P})\|\boldsymbol{\mu}\|^{2} \leq U(\boldsymbol{\mu}) \leq \lambda_{\max }(\boldsymbol{P})\|\boldsymbol{\mu}\|^{2}, \\
\sum_{i=1}^{2} \frac{\partial U}{\partial \mu_{\boldsymbol{t}}}\left(\mu_{i+1}-a_{i} \mu_{i}\right)-\frac{\partial U}{\partial \mu_{3}} a_{3} \mu_{1}= \\
=-\boldsymbol{\mu}^{T} \boldsymbol{\mu}=-\|\boldsymbol{\mu}\|^{2}=-W(\boldsymbol{\mu}), \\
\left|\frac{\partial U}{\partial \mu_{3}}\right| \leq\left\|\frac{\partial U}{\partial \boldsymbol{\mu}}\right\|=\left\|2 \boldsymbol{\mu}^{T} \boldsymbol{P}\right\| \leq 2\|\boldsymbol{P}\|\|\boldsymbol{\mu}\|=2 \lambda_{\max }(\boldsymbol{P})\|\boldsymbol{\mu}\|,
\end{gathered}
$$

where $\lambda_{\text {min }}(\boldsymbol{P}), \lambda_{\text {max }}(\boldsymbol{P})$ denote the maximum eigenvalue and minimum eigenvalue of matrix $\boldsymbol{P}$, respectively, $\|\bullet\|$ is the Euclidean norm.

Let

$$
\begin{gathered}
e_{i}(t)=f_{i_{-} x}-f_{i_{-} x}, \quad i=1,2,3, \\
\mu_{i}(t)=e_{i}(b t) / b^{3-i}, \quad i=1,2,3 .
\end{gathered}
$$

Direct derivation $\boldsymbol{\mu}=\left(\begin{array}{lll}\mu_{1} & \mu_{2} & \mu_{3}\end{array}\right)^{T}$ satisfies the following error system:

$$
\left\{\begin{array}{l}
\dot{\mu}_{1}=\mu_{2}-f a l\left(\mu_{1}, \partial_{1}, \delta\right), \mu_{1}(0)=e_{1}(0) / b^{2}, \\
\dot{\mu}_{2}=\mu_{3}-f a l\left(\mu_{2}, \partial_{1}, \delta\right), \mu_{2}(0)=e_{2}(0) / b \\
\dot{\mu}_{3}=-\operatorname{fal}\left(\mu_{3}, \partial_{2}, \delta\right)+b \square t, \mu_{3}(0)=e_{3}(0) .
\end{array}\right.
$$

Considering the derivative of $U(\boldsymbol{\mu}(t))$ along (31) with respect to $t$, we can deduce that

$$
\begin{aligned}
\frac{d U(\boldsymbol{\mu}(t))}{d t} & =\sum_{i=1}^{2} \frac{\partial U}{\partial \mu_{i}}\left(\mu_{i+1}-f a l\left(\mu_{1}, \partial_{i}, \delta\right)\right)- \\
& -\frac{\partial U}{\partial \mu_{3}} f a l\left(\mu_{1}, \partial_{2}, \delta\right)+\frac{\partial U}{\partial \mu_{3}} b \sqsubset t \leq-W(\boldsymbol{\mu})+b \rho\|\boldsymbol{\mu}\| \leq \\
& \leq-\frac{\lambda_{3}}{\lambda_{2}} U(\boldsymbol{\mu})+\frac{1}{\sqrt{\lambda_{1}}} b \rho \sqrt{U(\boldsymbol{\mu})},
\end{aligned}
$$

where $\lambda_{1}, \lambda_{2}, \lambda_{3}, \rho$ are the constants that can make the equation exist. According to the definitions of functions $U$ and $W$, we can know that there are constants of this type.

From (32), it can be obtained that

$$
\frac{d \sqrt{U(\boldsymbol{\mu}(t))}}{d t} \leq-\frac{\lambda_{3}}{\lambda_{2}} \frac{\sqrt{U(\boldsymbol{\mu})}}{2}+\frac{1}{2 \sqrt{\lambda_{1}}} b \rho .
$$

$$
\begin{aligned}
\|\boldsymbol{\mu}\| \leq \sqrt{\frac{U(\boldsymbol{\mu})}{\lambda_{1}}} & \leq \frac{\sqrt{\lambda_{1} U(\boldsymbol{\mu}(0))}}{\lambda_{1}} e^{-\frac{\lambda_{3}}{2 \lambda_{2}} t}+ \\
& +\frac{b \rho}{2 \lambda_{1}} \int_{0}^{t} e^{-\frac{\lambda_{3}}{2 \lambda_{2}}(t-s)} d s .
\end{aligned}
$$

Combined with (28), it can be concluded that

$$
\begin{aligned}
\left|e_{i}(t)\right| & =b^{3-i}\left|\mu_{i}\left(\frac{t}{b}\right)\right| \leq b^{3-i}\left\|\boldsymbol{\mu}\left(\frac{t}{b}\right)\right\| \leq \\
& \leq b^{3-i}\left[\frac{\sqrt{\lambda_{1} U(\boldsymbol{\mu}(0))}}{\lambda_{1}} e^{-\frac{\lambda_{3}}{2 \lambda_{2} b} t}+\frac{b \rho}{2 \lambda_{1}} \int_{0}^{\frac{t}{b}} e^{-\frac{\lambda_{3}}{2 \lambda_{2} b}(t-s)} d s\right]
\end{aligned}
$$

According to (35), when $b \rightarrow 0, e_{i}(t) \rightarrow 0$, the observer is stable.

\section{Simulations}

This section verifies the feasibility of the proposed control strategy in the presence of random disturbances. First, the dynamic simulation model of PMSA is established in ADAMS. The moment of inertia of PMSA is measured as seen in (36):

$$
\left\{\begin{array}{l}
I_{x}=I_{z}=0.01548\left(\mathrm{Kg} \bullet \mathrm{m}^{2}\right), \\
I_{y}=0.01571\left(\mathrm{Kg} \bullet \mathrm{m}^{2}\right) .
\end{array}\right.
$$

Let the system sampling time parameter $h=0.01$, tracking factor $\delta=10$, NESO parameters $b=\left[\begin{array}{lll}0.01 & 0.01 & 0.01\end{array}\right]$, and set the desired trajectory

$$
\boldsymbol{\theta}=\left[\begin{array}{lll}
\alpha & \beta & \gamma
\end{array}\right]^{T}=\left[\begin{array}{lll}
10 \sin (\pi t) & 10 \cos (\pi t) & 5 t
\end{array}\right]^{T} .
$$

Also, the model error is set as seen in (38)

$$
\Delta(\boldsymbol{\theta}, \dot{\boldsymbol{\theta}})=0.25 M(\boldsymbol{\theta})+0.25 \boldsymbol{C}(\boldsymbol{\theta}, \dot{\boldsymbol{\theta}}) .
$$

Add a random disturbance

$$
d=\sin (h t)+h \text {. }
$$

At the same time, two groups of comparative simulation are added, which are the linear extended state observer (LESO) and traditional Proportional Derivative (PD) control. The parameters are set as follows.

PD control: $K_{p}=0.1, K_{d}=0.02$.

The structures of LESO and TSMC-F are shown in references [25] and [32], respectively. Setting parameters:

$$
\begin{gathered}
\left\{\begin{array}{l}
\boldsymbol{w}_{\boldsymbol{0}}=\operatorname{diag}\left[\begin{array}{lll}
100 & 100 & 100
\end{array}\right], \\
\boldsymbol{w}_{\boldsymbol{c}}=\operatorname{diag}\left[\begin{array}{lll}
110 & 110 & 110
\end{array}\right],
\end{array}\right. \\
\left\{\begin{array}{l}
(p, q)=(9,5), \varepsilon=0.01, \\
\boldsymbol{\Gamma}=\operatorname{diag}\left[\begin{array}{lll}
0.35 & 0.35 & 0.35
\end{array}\right], \\
\boldsymbol{K}=\operatorname{diag}\left[\begin{array}{lll}
50 & 50 & 50
\end{array}\right] .
\end{array}\right.
\end{gathered}
$$

From the definition of (27) and $U$ and $W$, it can be seen that 
The tracking effect of the three axes is shown in Figs. 3-5, and the tracking error is shown in Figs. 6-8. Table I shows the mean square deviation of different angles under different tracking methods.

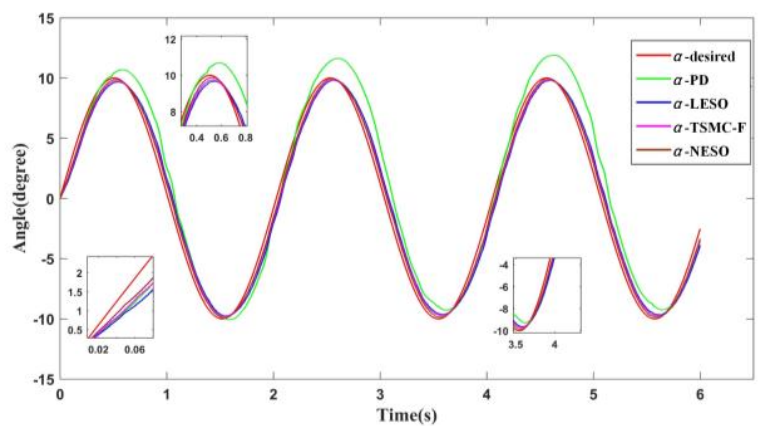

Fig. 3. Comparison of the tracking effect of $\alpha$ angle.

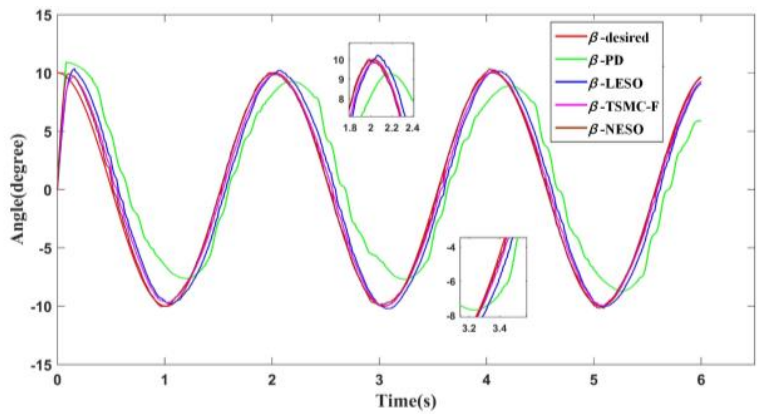

Fig. 4. Comparison of the tracking effect of $\beta$ angle.

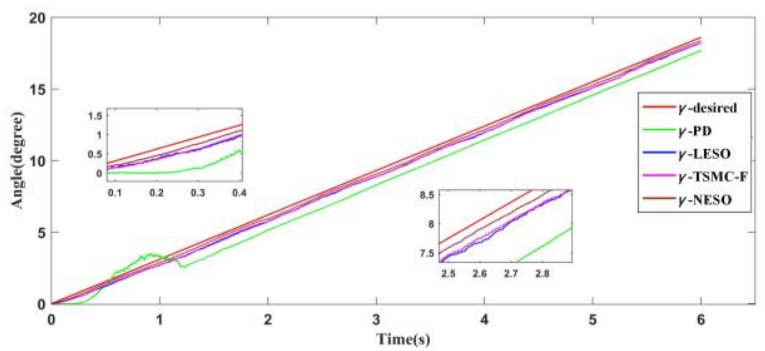

Fig. 5. Comparison of the tracking effect of $\gamma$ angle.

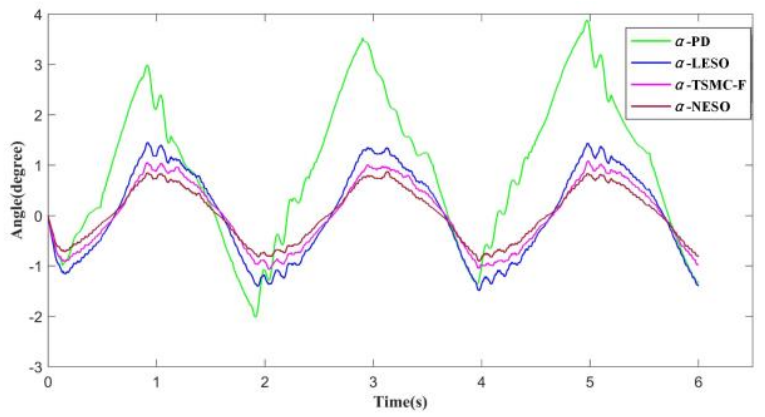

Fig. 6. Comparison of the tracking errors of $\alpha$ angle.

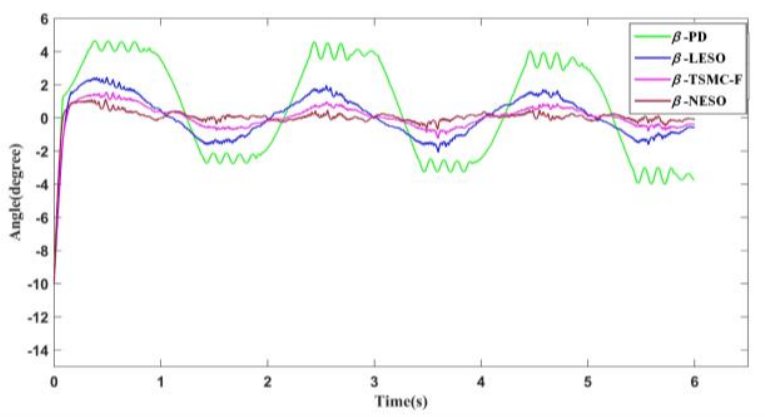

Fig. 7. Comparison of the tracking errors of $\beta$ angle.

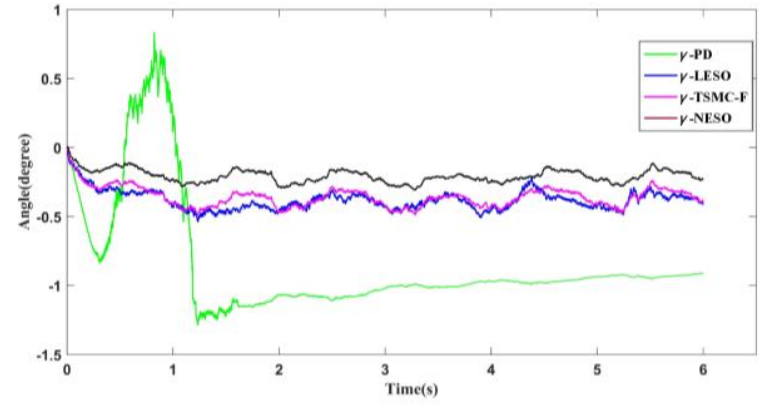

Fig. 8. Comparison of the tracking errors of $\gamma$ angle.

The simulation results show that, compared to the ordinary PD control, the control errors of the three axes under NESO are reduced by $76.5 \%, 84.5 \%$, and $76.5 \%$, respectively. Compared to conventional LESO control, the NESO control errors of the three axes are reduced by $38.9 \%, 65.7 \%$, and $44.4 \%$, respectively. In addition, compared to the TSMC-F control, the control errors of the three axes under NESO are reduced by $22.8 \%, 30.7 \%$, and $64.4 \%$, respectively. The simulation results show that the NESO control can effectively reduce the errors caused by internal coupling, friction, and uncertainty, and can provide better control accuracy than other control methods.

TABLE I. MEAN SQUARE ERRORS VERSUS THE DIFFERENT

\begin{tabular}{|c|c|c|c|c|}
\hline Items & PD & LESO & TSMC-F & NESO \\
\hline $\begin{array}{c}\text { Errors on } \\
\text { angle } \boldsymbol{\alpha}\end{array}$ & 1.7133 & 0.8970 & 0.6952 & 0.5339 \\
\hline $\begin{array}{c}\text { Errors on } \\
\text { angle } \boldsymbol{\beta}\end{array}$ & 3.0317 & 1.3541 & 0.9655 & 0.6688 \\
\hline $\begin{array}{c}\text { Errors on } \\
\text { angle } \boldsymbol{\gamma}\end{array}$ & 0.8875 & 0.1503 & 0.1288 & 0.0459 \\
\hline
\end{tabular}

\section{EXPERIMENTS}

The experimental device is shown in Fig. 9. It consists of a PMSA, a upper computer, a MEMS sensor, a driving circuit, and a power supply. The detailed structure of PMSA used in this platform is described in Section II.

In this paper, an MEMS wireless position sensor is installed on the output shaft of PMSA, which can communicate with the upper computer controller through Bluetooth serial communication. The position information detected by the MEMS sensor is used as feedback to control. The accuracy of position detection of PMSA used in this paper can be seen in [35]. The operation principle of the experimental platform is to calculate the current required by each coil after the upper computer gets the Desired position and the real-time position measured by the MEMS sensor, and then the current source will power up the driving circuit to make the PMSA move towards the new position. The driving circuit consists of 24 ARMs, each of which controls the current of a single coil of PMSA.

Set the desired trajectory to

$$
\boldsymbol{\theta}=\left[\begin{array}{lll}
\alpha & \beta & \gamma
\end{array}\right]^{T}=[10 \sin (\pi t) 10 \cos (\pi t) e t]^{T},
$$

where $e$ is the natural logarithm.

In this section, the PD and linear observer are also set in. The parameters of various control strategies are set in the same way as in simulation. 


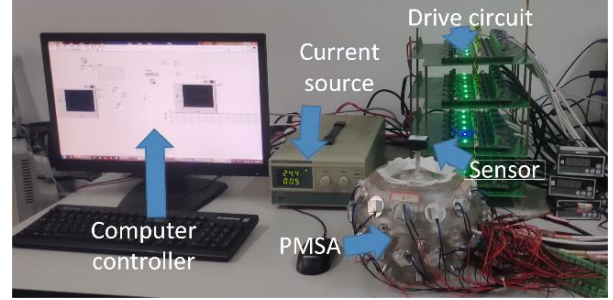

Fig. 9. Experimental platform.

The tracking effect of the three axes is shown in Figs. 10-12, and the tracking error is shown in Figs. 13-15. Table II shows the mean square deviation of different angles under different tracking methods.

It can be seen from the figure that the control errors of three axes under the control of NESO are reduced by $53.1 \%$, $78.7 \%$, and $53.2 \%$, respectively, compared to the ordinary PID control. Compared to the conventional LESO control, the control errors of the three axes are reduced by $23.2 \%$, $62.2 \%$, and $33.3 \%$, respectively. In addition, the control errors of three axes under the control of NESO are reduced by $17.4 \%, 44.7 \%$, and $50.9 \%$, respectively, compared to the TSMC-F control. The experimental results show that the control method used in this paper can effectively suppress the influence of uncertain disturbance, friction, and other factors. It has high control precision, fast tracking speed, good dynamic performance, robustness, and decoupling.

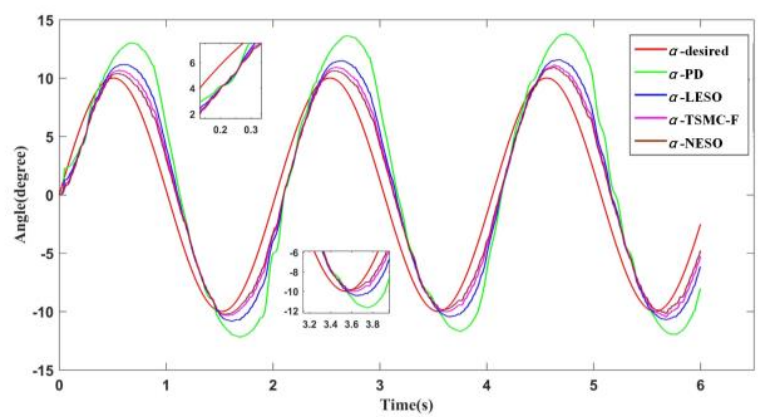

Fig. 10. Comparison of the tracking effect of $\alpha$ angle.

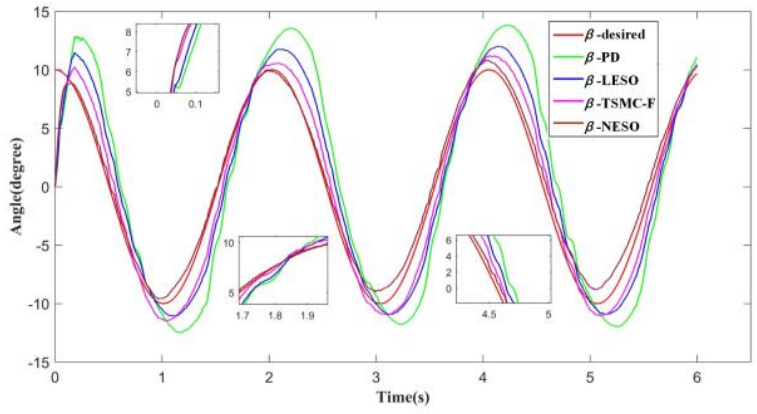

Fig. 11. Comparison of the tracking effect of $\beta$ angle.

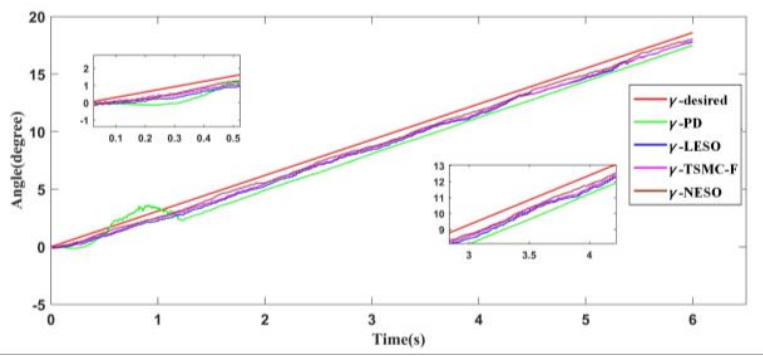

Fig. 12. Comparison of the tracking effect of $\gamma$ angle.

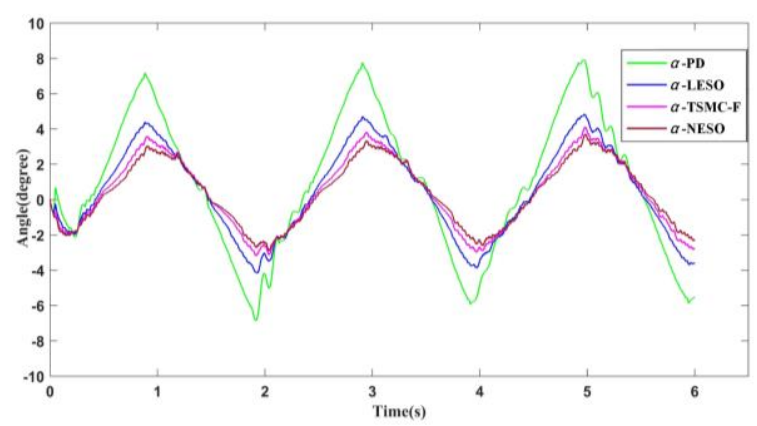

Fig. 13. Comparison of the tracking errors of $\alpha$ angle.

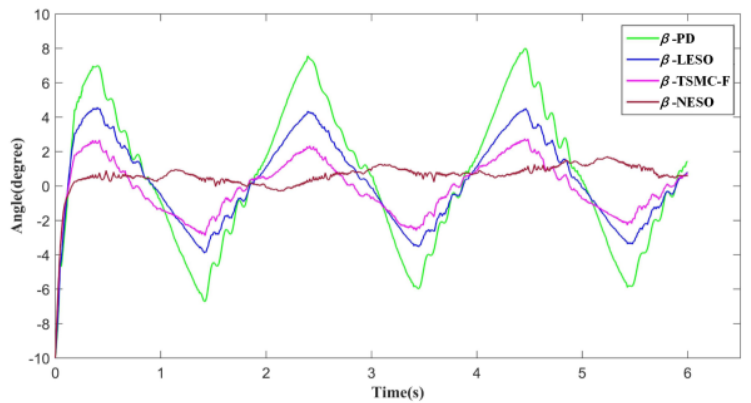

Fig. 14. Comparison of the tracking errors of $\beta$ angle.

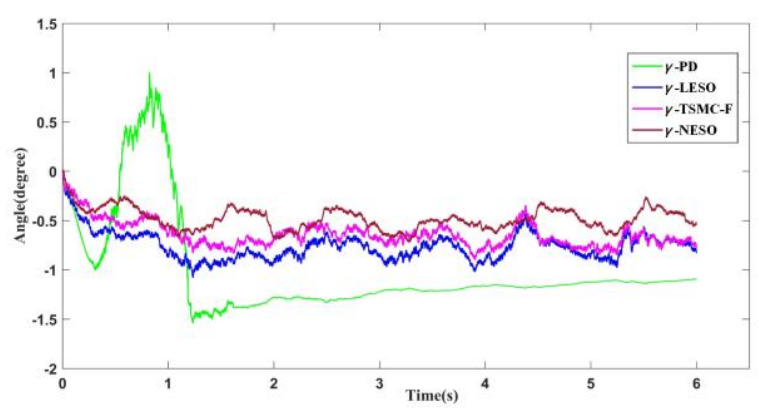

Fig. 15. Comparison of the tracking errors of $\gamma$ angle.

TABLE II. MEAN SQUARE ERRORS VERSUS THE DIFFERENT

\begin{tabular}{|c|c|c|c|c|}
\hline \multicolumn{5}{|c}{ ANGLE. } \\
\hline $\begin{array}{c}\text { Items } \\
\text { anrors on } \boldsymbol{\alpha}\end{array}$ & 3.9303 & 2.5331 & 2.2353 & 1.8468 \\
\hline $\begin{array}{c}\text { Errors on } \\
\text { angle } \boldsymbol{\beta}\end{array}$ & 4.0415 & 2.5118 & 1.8409 & 1.0183 \\
\hline $\begin{array}{c}\text { Errors on } \\
\text { angle } \boldsymbol{\gamma}\end{array}$ & 1.2679 & 0.6011 & 0.4315 & 0.2120 \\
\hline
\end{tabular}

Obviously, for PD control, it does not consider the nonlinear factors of the model, so it is difficult to ensure that the controlled object has good dynamic performance. The LESO control uses the linear gain instead of the actual nonlinear gain, which cannot guarantee the excellent control accuracy. TSMC-F control is difficult to balance control accuracy and chattering in the parameter adjustment process. Therefore, the use of NESO control will have a better effect to a certain extent.

\section{CONCLUSIONS}

For the trajectory tracking control system of PMSA, an ADRC scheme based on NESO is proposed in this paper. The NESO is used as the core part of the decoupling, and the coupling problem between different rotation angles is 
transformed into the disturbance problem by using the NESO. For the problems of low accuracy and poor dynamic and static performance in general controllers, the contributions of this paper are as follows:

1. It is worth noting that the improved observer has a nonlinear structure and contains only a few parameters, so the control of PMSA is more efficient.

2. The simulation and experimental results verify the effectiveness and correctness of the control scheme, which can effectively eliminate the influence of cross coupling and external uncertain interference in the PMSA. 3. In addition, the static and dynamic decoupling performance has been greatly improved, which is robust to the random disturbance.

It provides a better choice for the following research of PMSA control and application, and it makes it possible for the spherical motor to replace the single axis motor in multi degree of freedom devices, such as manipulator and satellite attitude, etc.

Although the nonlinear extended observer simplifies the process of parameter adjustment. However, under the control strategy proposed in this paper, it is still necessary to study further how to select the optimal nonlinear function and the value of the optimal parameters. In addition, the MIMO control system with better control effect can also be considered in the future.

\section{CONFLICTS OF INTEREST}

Authors declare that they have no conflict of interest.

\section{REFERENCES}

[1] M.-S. Huang, Y. L. Hsu, and R. F. Fung, "Minimum-energy point-to-point trajectory planning for a motor-toggle servomechanism", IEEE/ASME Transactions on Mechatronics, vol 17, no. 2, pp. 337-343, 2012. DOI: 10.1109/TMECH.2010.2103366.

[2] D. E. Ezenekwe and K. M. Lee, "Design of air bearing system for fine motion application of multi-DOF spherical actuator", in Proc. of 1999 IEEE/ASME International Conference on Advanced Intelligent Mechatronics (Cat. No. 99TH8399), 1999, pp. 812-818. DOI: 10.1109/AIM.1999.803277.

[3] H. Son and K.-M. Lee, "Open-loop controller design and dynamic characteristics of a spherical wheel motor", IEEE Transactions on Industrial Electronics, vol. 57, no. 10, pp. 3475-3482, 2010. DOI: 10.1109/TIE.2009.2039454.

[4] F. Chai, L. Gan, and L. Chen, "A novel tiered type permanent magnet spherical motor and its rotor orientation measurement principle", IEEE Access, vol. 8, pp. 15303-15312, 2020. DOI: 10.1109/ACCESS.2020.2966786.

[5] J. F. P. Fernandes and P. J. C. Branco, "The shell-like spherical induction motor for low-speed traction: Electromagnetic design, analysis, and experimental tests", IEEE Transactions on Industrial Electronics, vol. 63, no. 7, pp. 4325-4335, Jul. 2016. DOI: 10.1109/TIE.2016.2535982.

[6] A. Ozdil and Y. Uzun, "Design and Analysis of a Rotor for a $22 \mathrm{~kW}$ Transversally Laminated Anisotropic Synchronous Reluctance Motor, Elektronika ir Elektrotechnika, vol. 27, no. 1, pp. 17-24, 2021.DOI 10.5755/j02.eie.29046.

[7] K. Takahara, K. Hirata, N. Niguchi, Y. Nishiura, and Y. Sakaidani, "Experimental evaluation of the static characteristics of multi-degree-of-freedom spherical actuators", IEEE Transactions on Magnetics, vol. 53, no. 11, pp. 1-5, art. no. 8205705, Nov. 2017. DOI: 10.1109/TMAG.2017.2717870.

[8] K.-M. Lee, J. Pei, and R. Roth, "Kinematic analysis of a three-degrees-of-freedom spherical wrist actuator", Mechatronics, vol. 4, no. 6, pp. 581-605, 1994. DOI: 10.1016/0957-4158(94)90026-4.

[9] K.-M. Lee, R. B. Roth, and Z. Zhou, "Dynamic modeling and control of a ball-joint-like variable-reluctance spherical motor", Journal of
Dynamic Systems, Measurement, and Control, vol. 118, no. 1, pp. 29-40, 1996. DOI: 10.1115/1.2801148.

[10] K. Lee and H. Son, "Torque model for design and control of a permanent spherical wheel motor", in Proc. of IEEE/ASME International Conference on Advanced Intelligent Mechatronics, 2005 , pp. 335-340. DOI: 10.1109/AIM.2005.1501013.

[11] M. Tsukano, K. Hirata, S. Maeda, and N. Niguchi, "Development of control method for outer-rotor spherical actuator", Electrical Engineering in Japan, vol. 194, no. 2, p. 5463, 2016. DOI: 10.1002/eej.22729

[12] K. Bai and K.-M. Lee, "Direct field-feedback control of a ball-joint-like permanent-magnet spherical motor", IEEE/ASME Transactions on Mechatronics, vol. 19, no. 3, pp. 975-986, Jun. 2014 DOI: 10.1109/TMECH.2013.2264565.

[13] H. Son and K.-M. Lee, "Control system design and input shape for orientation of spherical wheel motor", Control Engineering Practice, vol. 24, pp. 120-128, 2014. DOI: 10.1016/j.conengprac.2013.11.013.

[14] S. Ilgen, A. Durdu, E. Gulbahce, and A. Cakan, "Optimal tuning of the SMC parameters for a two-link manipulator co-simulation control", Elektronika ir Elektrotechnika, vol. 27, no. 5, pp. 4-10, 2021. DOI: 10.5755/j02.eie.28954.

[15] S.-H. Rhyu, H.-U. Shin, M.-S. Kim, and B.-I. Kwon, "Dynamic analysis of double excited 3-DOF motor modeling using equivalent magnetic circuit", Journal of Electrical Engineering \& Technology, vol. 10, no. 3, pp. 958-964, 2015. DOI: 10.5370/JEET.2015.10.3.958.

[16] X. Guo, K. Pan, Q. Wang, and Y. Wen, "Robust adaptive sliding-mode control of a permanent magnetic spherical actuator with delay compensation", IEEE Access, vol. 8, pp. 128096-128105, 2020. DOI: 10.1109/ACCESS.2020.3008152.

[17] L. Zhang, W. Chen, J. Liu, and C. Wen, "A robust adaptive iterative learning control for trajectory tracking of permanent-magnet spherical actuator", IEEE Transactions on Industrial Electronics, vol. 63, no. 1, pp. 291-301, Jan. 2016. DOI: 10.1109/TIE.2015.2464186.

[18] W. Chen, L. Zhang, L. Yan, and J. Liu, "Design and control of a three degree-of-freedom permanent magnet spherical actuator", Sensors and Actuators A: Physical, vol. 180, pp. 75-86, 2012. DOI: 10.1016/j.sna.2012.04.010

[19] H. Li, Z. Song, C. Guo et al., "Control for trajectory tracking of permanent magnet spherical motor based on computed torque method", Journal of Tianjin University Science and Technology, vol. 46, no. 11, pp. 991-997, 2013. DOI: 10.11784/tdxb20131107.

[20] X. Guo, S. Li, Q. Wang, Y. Wen, and N. Gong, "Dynamic analysis and current calculation of a permanent magnet spherical motor for point-to-point motion", IET Electric Power Applications, vol. 13, no. 4, pp. 426-434, 2019. DOI: 10.1049/iet-epa.2018.5149.

[21] C. Xia, C. Guo, and T. Shi, "A neural-network-identifier and fuzzy-controller-based algorithm for dynamic decoupling control of permanent-magnet spherical motor", IEEE Transactions on Industrial Electronics, vol. 57, no. 8, pp. 2868-2878, Aug. 2010. DOI 10.1109/TIE.2009.2036030

[22] X. Guo, Q. Wang, G. Li et al., "Adaptive tracking control using partitioned RBFNN in permanent magnet spherical motor system", Journal of Computational Information Systems, vol. 10, no. 7, pp. 2711-2718, 2014. DOI: 10.12733/jcis9614.

[23] L. Yan, L. Zhang, B. Zhu, J. Zhang, and Z. Jiao, "Single neural adaptive controller and neural network identifier based on PSO algorithm for spherical actuators with 3D magnet array", Review of Scientific Instruments, vol. 88, no. 10, p. 105001, 2017. DOI: 10.1063/1.5004677.

[24] H. Li, G. Li, Q. Wang, B. Ju, and Y. Wen, "Friction torque field distribution of a permanent-magnet spherical motor based on multi-physical field coupling analysis", IET Electric Power Applications, vol. 15, no. 8, pp. 1045-1055, 2021. DOI 10.1049/elp2.12096.

[25] K. Bai, H. Yan, and K.-M. Lee, "Robust control of a spherical motor in moving frame", Mechatronics, art. no. 102548, 2021. DOI: 10.1016/j.mechatronics.2021.102548.

[26] J. Han, "From PID to active disturbance rejection control", IEEE Transactions on Industrial Electronics, vol. 56, no. 3, pp. 900-906, 2009. DOI: 10.1109/TIE.2008.2011621.

[27] Y. X. Su, B. Y. Duan, and Y. F. Zhang, "Auto disturbance rejection motion control for direct-drive motors", in Proc. of IEEE 2002 28th Annual Conference of the Industrial Electronics Society, IECON 02, 2002, pp. 2073-2078, vol. 3. DOI: 10.1109/IECON.2002.1185292.

[28] Y. X. Su, C. H. Zheng, and B. Y. Duan, "Automatic disturbances rejection controller for precise motion control of permanent-magnet synchronous motors", IEEE Transactions on Industrial Electronics, 
vol. 52, no. 3, pp. 814-823, Jun. 2005. DOI: 10.1109/TIE.2005.847583.

[29] S. Li, X. Yang, and D. Yang, “Active disturbance rejection control for high pointing accuracy and rotation speed", Automatica, vol. 45, no. 8, pp. 1854-1860, 2009. DOI: 10.1016/j.automatica.2009.03.029.

[30] X. Hai et al., "Mobile robot ADRC with an automatic parameter tuning mechanism via modified pigeon-inspired optimization", IEEE/ASME Transactions on Mechatronics, vol. 24, no. 6, pp. 2616-2626, Dec. 2019. DOI: 10.1109/TMECH.2019.2953239.

[31] X. Gong, Y. Tian, Y. Bai, and C. Zhao, "Trajectory tacking control of a quad-rotor based on active disturbance rejection control", in Proc. of 2012 IEEE International Conference on Automation and Logistics, 2012, pp. 254-259. DOI: 10.1109/ICAL.2012.6308207.

[32] J. Liu, H. Deng, W. Chen, and S. Bai, "Robust dynamic decoupling control for permanent magnet spherical actuators based on extended state observer", IET Control Theory and Applications, vol. 11, no. 5, pp. 619-631, 2017. DOI: 10.1049/iet-cta.2016.0551.

[33] Z. Qian, Q. Wang, L. Ju, A. Wang, and J. Liu, "Torque modeling and control algorithm of a permanent magnetic spherical motor", in Proc. of 2009 International Conference on Electrical Machines and Systems, 2009, pp. 1-6. DOI: 10.1109/ICEMS.2009.5382656.

[34] Q. Wang and K. Xia, "Simulation of current control for a permanent magnet spherical stepper motor", in Proc. of 2007 International Conference on Electrical Machines and Systems (ICEMS), 2007, pp. 547-551. DOI: 10.1109/ICEMS12746.2007.4412023.

[35] Y. Rong, Q. Wang, S. Lu, G. Li, Y. Lu, and J. Xu, "Improving attitude detection performance for PMSAs using a MEMS inertial measurement sensor", IET Electric Power Applications, vol. 13, no. 2, pp. 198-205, 2019. DOI: 10.1049/iet-epa.2018.5195.

This article is an open access article distributed under the terms and conditions of the Creative Commons Attribution 4.0 (CC BY 4.0) license (http://creativecommons.org/licenses/by/4.0/) 